\title{
Theory for Magnetism and Triplet Superconductivity in LiFeAs
}

\author{
P. M. R. Brydon, ${ }^{1, \text { | }}$ Maria Daghofer, ${ }^{2}$, Carsten Timm, ${ }^{1}$ and Jeroen van den Brink ${ }^{2}$ \\ ${ }^{1}$ Institut für Theoretische Physik, Technische Universität Dresden, 01062 Dresden, Germany \\ ${ }^{2}$ IFW Dresden, P.O. Box 270116, 01171 Dresden, Germany
}

(Dated: October 26, 2018)

\begin{abstract}
Superconducting pnictides are widely found to feature spin-singlet pairing in the vicinity of an antiferromagnetic phase, for which nesting between electron and hole Fermi surfaces is crucial. LiFeAs differs from the other pnictides by (i) poor nesting properties and (ii) unusually shallow hole pockets. Investigating magnetic and pairing instabilities in an electronic model that incorporates these differences, we find antiferromagnetic order to be absent. Instead we observe almost ferromagnetic fluctuations which drive an instability toward spin-triplet $p$-wave superconductivity.

PACS numbers: 74.70.Xa, 74.20.Rp, 74.20.Mn
\end{abstract}

\section{INTRODUCTION}

The family of pnictide superconductors has generated large interest during the last two years, because their superconductivity (SC) is generally thought to be unconventional, i.e., not due to phonons, and because some pnictides have high transition temperatures reminiscent of cuprates. The details of the pairing are far from being clarified, and may vary significantly between the different families; for two recent reviews see Refs. 112. A plausible scenario for the pairing mechanism involves exchange of virtual spin fluctuations, as SC is often observed in the vicinity of an antiferromagnetic (AF) spin-densitywave (SDW) state. In the 1111 and 122 families, the SDW is apparently driven by excellent nesting of electron and hole Fermi pockets. When doping reduces the nesting, SDW order is weakened, but AF fluctuations remain and are believed to provide the pairing for the Cooper pairs ${ }^{314}$ Together with the high transition temperatures observed in some pnictides, such a scenario raises hopes that pnictides might shed light onto similarities - or differences - between spin-fluctuation superconductors like heavy-fermion systems, with typically lower transition temperatures, $\sqrt{56}$ and the more enigmatic cuprates.

The recently-discovered pristine superconductor LiFeAs differs in key respects from the most commonly studied pnictide families. Doping is not necessary for superconductivity,,$^{7 / 9}$ the nesting between hole and electron pockets is rather poor and consequently an SDW is not observed. Moreover, compared to other pnictide families, LiFeAs has much shallower hole pockets around the center of the Brillouin zone ${ }^{9}$ 11 It was pointed out that the flat top of these pockets implies a large density of states, ${ }^{[9}$ which one can expect to promote ferromagnetic (FM) fluctuations. Indeed, recent nuclear magnetic resonance (NMR) measurements indicate a relatively high susceptibility within the $a-b$ planes. Remarkably, the Knight shift does not change at the superconducting transition for magnetic fields perpendicular to the $c$ axis, $\frac{12}{, 2}$ which is evidence for triplet superconductivity. This discovery relates a member of the pnictide family to yet another family with unconventional SC, the ruthenates 13

In this paper we model the distinguishing electronic features of LiFeAs, i.e., the absence of nesting and the presence of shallow hole pockets. We examine the magnetic properties of this Hamiltonian by combining a mean-field approximation and the random-phase approximation (RPA). We find that the magnetic fluctuations are dominated by almost FM processes across the smaller hole pocket. We proceed to investigate what types of Cooper pairs can be stabilized by the spin and charge fluctuations. We use the same approach that has been used to treat other pnictides, and which yields AF order and singlet pairing in the 1111 and 122 families $\frac{14}{17} \mathrm{In}$ the case of LiFeAs, however, we find that the almost FM fluctuations can drive triplet $p$-wave superconductivity. Throughout, we discuss the connection of our work to experimental results.

The possibility of triplet pairing was already discussed in the early days of pnictide research. The degeneracy of the $x z$ and $y z$ orbitals that dominate the Fermi surface (FS) permits spin-triplet and orbital-singlet pairs with (nodal) $s$ - or $d$-wave-like character $\frac{18] 23}{23}$ Triplets with $p$-wave symmetry, stabilized by large Hund's rule coupling, were reported for a three-band model and involved mostly the electron pockets. ${ }^{24}$ The mechanism proposed in this paper, however, is very different and specific to LiFeAs, originating from the high density of states due to the rather flat 'roofs' of the shallow hole pockets.

\section{MODEL}

In order to capture the most relevant states around the FS, we model the two concentric hole pockets and the two electron pockets, which can be achieved using three orbitals per iron ion. We use a one-iron unit cell, with $\mathbf{k}$ running over $-\pi<k_{x}, k_{y} \leq \pi$ in the corresponding extended Brillouin zone, and the hole pockets are then around $\Gamma=(0,0)$, while the electron pockets are found at $X=(\pi, 0)$ and $Y=(0, \pi)$. The three orbitals with the largest weight in these three pockets are the $x z, y z$, and $x y$ orbitals, denoted by indices 1,2 and 3 . The momentum-dependent non-interacting Hamiltonian 

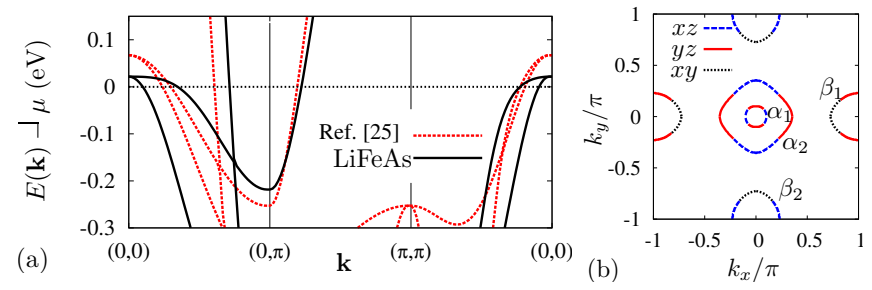

FIG. 1: (Color online) (a) Band structure of (dashed lines) the three-band model Eq. (1) from Ref. 25] and (solid lines) the model for LiFeAs. (b) Fermi surface and dominant orbitals for the LiFeAs model; $\alpha_{1}$ and $\alpha_{2}$ are hole pockets, $\beta_{1}$ and $\beta_{2}$ are electron pockets. Parameters: $t_{1}=0.02, t_{2}=0.12, t_{3}=0.02$, $t_{4}=-0.046, t_{5}=0.2, t_{6}=0.3, t_{7}=-0.15, t_{8}=-t_{7} / 2$, $t_{9}=-0.06, t_{10}=-0.03, t_{11}=0.014, \Delta_{x y}=1, \mu=0.338$. The filling is four electrons per site, energies are in $\mathrm{eV}$.

is given by

$$
H_{0}(\mathbf{k})=\sum_{\mathbf{k}, \sigma, \mu, \nu} T^{\mu, \nu}(\mathbf{k}) d_{\mathbf{k}, \mu, \sigma}^{\dagger} d_{\mathbf{k}, \nu, \sigma},
$$

where $d_{\mathbf{k}, \mu, \sigma}^{\dagger}\left(d_{\mathbf{k}, \mu, \sigma}\right)$ creates (annihilates) an electron in orbital $\mu$ with momentum $\mathbf{k}$ and spin $\sigma$. The elements of the hopping matrix $T^{\mu, \nu}(\mathbf{k})$ are

$$
\begin{aligned}
T^{11 / 22}= & 2 t_{2 / 1} \cos k_{x}+2 t_{1 / 2} \cos k_{y}+4 t_{3} \cos k_{x} \cos k_{y} \\
& \pm 2 t_{11}\left(\cos 2 k_{x}-\cos 2 k_{y}\right)-\mu \\
T^{33}= & \Delta_{x y}+2 t_{5}\left(\cos k_{x}+\cos k_{y}\right)+4 t_{6} \cos k_{x} \cos k_{y} \\
& +2 t_{9}\left(\cos 2 k_{x}+\cos 2 k_{y}\right) \\
& +4 t_{10}\left(\cos 2 k_{x} \cos k_{y}+\cos k_{x} \cos 2 k_{y}\right)-\mu \\
T^{12}= & T^{21}=-4 t_{4} \sin k_{x} \sin k_{y} \\
T^{13}= & \bar{T}^{31}=2 i t_{7} \sin k_{x}+4 i t_{8} \sin k_{x} \cos k_{y} \\
T^{23}= & \bar{T}^{32}=2 i t_{7} \sin k_{y}+4 i t_{8} \sin k_{y} \cos k_{x}
\end{aligned}
$$

where a bar on top of a matrix element denotes the complex conjugate. The parameters are given in the caption of Fig. 1 .

The one-particle bands defined by this kinetic energy are shown in Fig. 1(a) for two parameterizations: one is the three-band model of Ref. 25, where only hopping to first and second neighbors is included. While this model already has rather shallow hole pockets compared to $a b$ initio bands for most pnictide compounds, it can reproduce spectral features of the AF phase observed in many undoped pnictides ${ }^{25 / 26}$ and has an instability to singlet pairing. 17 The second more refined model makes use of longer-range hoppings in order to describe the bands of LiFeAs more closely. The two hole pockets, which have mostly $x z$ and $y z$ character and are degenerate at $\Gamma$, are far shallower than the electron pockets and have very different radii in accordance with ARPES findings and $a b$ initio density functional calculations. 11 Both models reflect the poor nesting of the electron and hole pockets in LiFeAs, see Fig. 1(b). As will be discussed later, the flat top of the hole pockets has a crucial impact

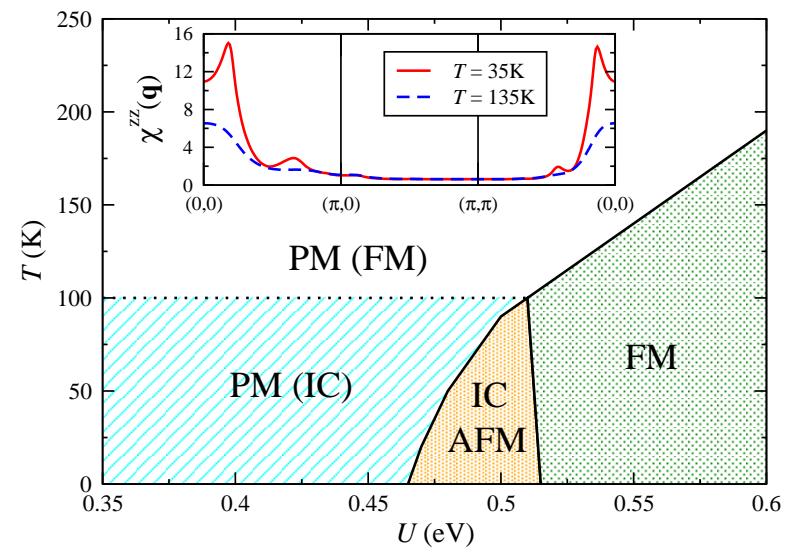

FIG. 2: (Color online) Magnetic phase diagram of the threeband model for LiFeAs. Inset: Magnetic susceptibility $\chi^{z z}(\mathbf{q})$ for $U=0.42 \mathrm{eV}$ and $T=35 \mathrm{~K}$ and $T=135 \mathrm{~K}$.

on the model properties; angle-resolved photo-emission (ARPES) data ${ }^{9}$ might suggest even flatter hole pockets.

We include all Coulomb interactions between electrons in the same Fe atom:

$$
\begin{aligned}
H_{I} & =U \sum_{\mathbf{i}, \nu} n_{\mathbf{i}, \nu, \uparrow} n_{\mathbf{i}, \nu, \downarrow}+V \sum_{\mathbf{i}, \nu \neq \mu} \sum_{\sigma, \sigma^{\prime}} n_{\mathbf{i}, \nu, \sigma} n_{\mathbf{i}, \mu, \sigma^{\prime}} \\
& -J \sum_{\mathbf{i}, \nu \neq \mu} \mathbf{S}_{\mathbf{i}, \nu} \cdot \mathbf{S}_{\mathbf{i}, \mu}+J^{\prime} \sum_{\mathbf{i}, \nu \neq \mu} d_{\mathbf{i}, \nu, \uparrow}^{\dagger} d_{\mathbf{i}, \nu, \downarrow}^{\dagger} d_{\mathbf{i}, \mu, \downarrow} d_{\mathbf{i}, \mu, \uparrow} .
\end{aligned}
$$

Here $n_{\mathbf{i}, \nu, \sigma}\left(\mathbf{S}_{\mathbf{i}, \nu}\right)$ is the number (spin) operator for the orbital $\nu$ on site $\mathbf{i}$. Invariance under rotation of the orbital degrees of freedom is ensured by setting $J=J^{\prime}$ and $V=$ $(2 U-5 J) / 4$. We only present results for $J=0.25 U$, but we have verified that other choices lead to similar results.

\section{MAGNETISM}

Following Ref. 27, we construct the magnetic phase diagram by first using a mean-field ansatz restricted to FM and two-site AF phases 28 . Unlike models of the 1111 and 122 pnictides, here only FM and PM phases are stable. ${ }^{27}$ The phase diagram is then refined by evaluating the static transverse spin susceptibility $\chi^{-+}(\mathbf{q})$ within the RPA. This yields two important results: firstly, negative values of $\chi^{-+}(\mathbf{q})$ signal an instability toward a magnetically ordered state (FM for $\mathbf{q}=0$, AF otherwise); secondly, in the PM phase the maximum of $\chi^{-+}(\mathbf{q})$ indicates the wavevector of the dominant spin fluctuations. In the PM phase $\chi^{-+}(\mathbf{q})=2 \chi^{z z}(\mathbf{q})$ is obtained by solving the Dyson equation $\hat{\chi}^{S}=\hat{\chi}^{(0)}+\hat{\chi}^{(0)} \hat{U}^{S} \hat{\chi}^{S}$ for the irreducible spin susceptibility $\hat{\chi}^{S[29}$. The non-zero matrix elements of $\hat{U}^{S}$ can be found in Refs. 14-16. The Lindhard function $\hat{\chi}^{(0)}$ is defined

$$
\begin{gathered}
\chi_{\nu, \nu^{\prime}, \mu, \mu^{\prime}}^{(0)}\left(\mathbf{q}, i \omega_{n}\right)=-\frac{1}{N} \sum_{\mathbf{k}} \sum_{j, j^{\prime}} \frac{n_{F}\left(E_{j, \mathbf{k}}\right)-n_{F}\left(E_{j^{\prime}, \mathbf{k}+\mathbf{q}}\right)}{E_{j, \mathbf{k}}-E_{j^{\prime}, \mathbf{k}+\mathbf{q}}-i \omega_{n}} \\
\times u_{j, \nu^{\prime}}(\mathbf{k}) u_{j, \mu}^{*}(\mathbf{k}) u_{j^{\prime}, \mu^{\prime}}(\mathbf{k}+\mathbf{q}) u_{j^{\prime}, \nu}^{*}(\mathbf{k}+\mathbf{q})
\end{gathered}
$$


where $\nu, \nu^{\prime}, \mu, \mu^{\prime}$ refer to the orbital, $n_{F}(E)$ is the Fermi function, and $E_{j, \mathbf{k}}$ are the eigenvalues of $H_{0}(\mathbf{k})$ [Eq. (1)]. The coefficients $u_{j, \nu}(\mathbf{k})$ transform the diagonalizing (band) annihilation operators $\gamma_{j, \mathbf{k}}$ (corresponding to $\left.E_{j, \mathbf{k}}\right)$ into the orbital basis, i.e. $d_{\nu, \mathbf{k}}=\sum_{j} u_{j, \nu}(\mathbf{k}) \gamma_{j, \mathbf{k}}$. The static transverse spin susceptibility is then written $\chi^{-+}(\mathbf{q})=\sum_{\nu, \mu} \chi_{\nu, \nu, \mu, \mu}^{S}(\mathbf{q}, \omega=0)$.

Figure 2 shows the phase diagram as a function of $U$ and temperature $T$. At low $U$, the model remains PM down to $T=0 \mathrm{~K}$, but the dominant fluctuations change from FM to incommensurate $(\mathrm{IC})$ at $T \approx 100 \mathrm{~K}$. As $U$ is increased, the IC fluctuations can drive a transition to an IC-AF state, while at higher $U$ a FM phase is realized. The wave vectors characterizing the IC fluctuations in the low- $T$ PM state lie on a ring-shaped feature centered at $\mathbf{q}=(0,0)$, as can be seen in the example plotted as the inset in Fig. 2. The radius of the ring is exactly twice the radius of the inner hole pocket, revealing that it originates from scattering diagonally across this $\mathrm{F} \$ 30$. This scattering is strongly favored because of the identical orbital composition of diagonally separated parts of the FS.

Experimentally, a relatively large susceptibility is observed parallel to the $a b$-plane, which slightly decreases with $T{ }^{12}$ While an increasing susceptibility is expected for dominant FM fluctuations, this observation is consistent with 'almost FM' IC fluctuations, where the weight observed at $(0,0)$ in $\chi^{z z}(\mathbf{q})$ no longer grows with lowering $T$ below the transition from FM to IC fluctuations. It may even slightly decrease as the ring feature becomes more pronounced, and our model shows such behavior at low temperature $T \lesssim 50 \mathrm{~K}$. These results indicate that $\mathrm{LiFeAs}$ is relatively near to a FM instability, which can be triggered by reducing the size of the inner hole pocket, e.g. by electron doping.

\section{PAIRING SYMMETRIES}

The strong spin fluctuations in the low- $T$ PM phase may drive the pairing of electrons. To determine the symmetry of a possible superconducting state, we employ a weak-coupling method due to Scalapino et al ${ }^{31}$ which has been widely used to study the pnictides 14

The pairing vertex due to the exchange of spin and charge fluctuations is obtained within the fluctuation exchange approximation. We have

$$
\begin{aligned}
\hat{\Gamma}^{S}\left(\mathbf{k}, \mathbf{k}^{\prime}, \omega\right)= & \frac{3}{2} \hat{U}^{S} \hat{\chi}^{S}\left(\mathbf{k}-\mathbf{k}^{\prime}, \omega\right) \hat{U}^{S}+\frac{1}{2} \hat{U}^{S} \\
& -\frac{1}{2} \hat{U}^{C} \hat{\chi}^{C}\left(\mathbf{k}-\mathbf{k}^{\prime}, \omega\right) \hat{U}^{C}+\frac{1}{2} \hat{U}^{C} \\
\hat{\Gamma}^{t}\left(\mathbf{k}, \mathbf{k}^{\prime}, \omega\right)= & -\frac{1}{2} \hat{U}^{S} \hat{\chi}^{S}\left(\mathbf{k}-\mathbf{k}^{\prime}, \omega\right) \hat{U}^{S}+\frac{1}{2} \hat{U}^{S} \\
& -\frac{1}{2} \hat{U}^{C} \hat{\chi}^{C}\left(\mathbf{k}-\mathbf{k}^{\prime}, \omega\right) \hat{U}^{C}+\frac{1}{2} \hat{U}^{C}
\end{aligned}
$$

for singlet and triplet pairing, respectively. Here $\hat{\chi}^{C}$ is the RPA irreducible charge susceptibility, which obeys
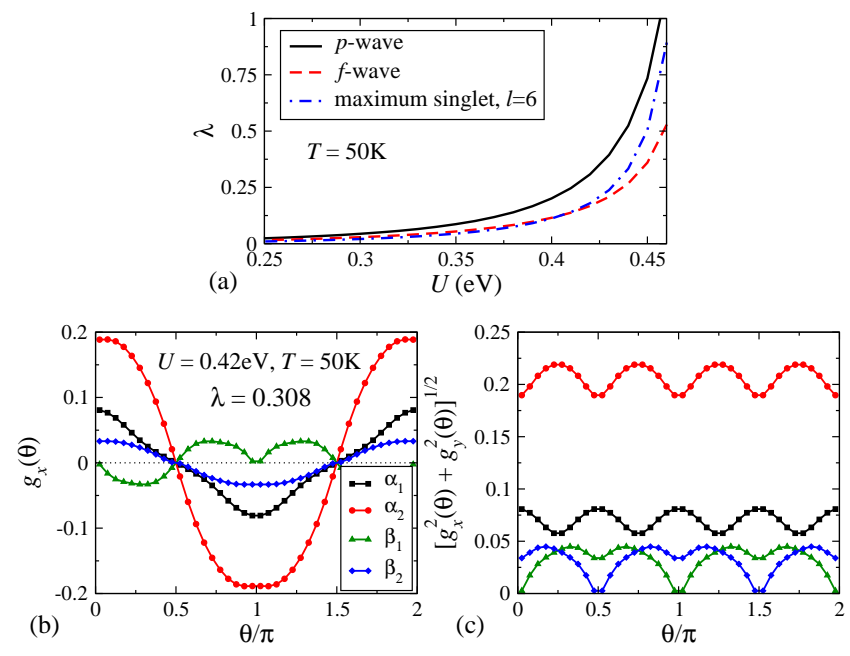

FIG. 3: (Color online) Triplet pairing. (a) Eigenvalues $\lambda$ of the leading gap symmetries as a function of $U$. (b) Gap around the Fermi pockets for the $p_{x}$-wave pairing, which is a member of the degenerate pair that gives the dominant triplet $p$-channel. The winding angle about the FSs $\theta$ is measured with respect to the $k_{x}$-axis, taken in the anti-clockwise direction. (c) Gap magnitude of pairing states combining the degenerate $p_{x}$ and $p_{y}$ solutions of Eq. (12). $\alpha_{1}, \alpha_{2}, \beta_{1}$, and $\beta_{2}$ refer to the FS pockets, see Fig. 1(b)

the Dyson equation $\hat{\chi}^{C}=\hat{\chi}^{(0)}-\hat{\chi}^{(0)} \hat{U}^{C} \hat{\chi}^{C}$. The nonzero elements of $\hat{U}^{C}$ are again found in Refs. 14 16.

Assuming that the dominant scattering occurs close to the FSs $\frac{14}{17}$, we describe the scattering of a Cooper pair from the state $(\mathbf{k},-\mathbf{k})$ on FS $C_{i}$ to the state $\left(\mathbf{k}^{\prime},-\mathbf{k}^{\prime}\right)$ on FS $C_{j}$ by the projected vertices

$$
\begin{aligned}
\Gamma_{i, j}^{\nu=s, t}\left(\mathbf{k}, \mathbf{k}^{\prime}\right)= & \sum_{\alpha, \beta, \gamma, \delta} u_{i, \gamma}(-\mathbf{k}) u_{i, \alpha}(\mathbf{k}) u_{j, \beta}^{*}\left(\mathbf{k}^{\prime}\right) u_{j, \delta}^{*}\left(-\mathbf{k}^{\prime}\right) \\
& \times \operatorname{Re}\left\{\Gamma_{\alpha, \beta, \gamma, \delta}^{\nu=s, t}\left(\mathbf{k}-\mathbf{k}^{\prime}, \omega=0\right)\right\}
\end{aligned}
$$

The superconducting gap on the FSs is written as $\Delta(\mathbf{k})=$ $\Delta g_{\nu}(\mathbf{k})$, where $g_{\nu}(\mathbf{k})$ is a dimensionless function describing the momentum dependence, and $\nu=s(t)$ denotes a singlet (triplet) state with even (odd) parity. $g_{\nu}(\mathbf{k})$ is obtained by solving the eigenvalue problem

$$
-\sum_{j} \oint_{C_{j}} \frac{d k_{\|}^{\prime}}{4 \pi^{2} v_{F, j}\left(\mathbf{k}^{\prime}\right)} \Gamma_{i j}^{\nu}\left(\mathbf{k}, \mathbf{k}^{\prime}\right) g_{\nu}\left(\mathbf{k}^{\prime}\right)=\lambda g_{\nu}(\mathbf{k})
$$

where $v_{F, i}(\mathbf{k})$ is the Fermi velocity and $\lambda$ is a dimensionless coupling strength. The gap function $g_{\nu}(\mathbf{k})$ with the largest $\lambda$ has the highest $T_{c}$, and hence fixes the symmetry of the pairing state. We use a $192 \times 192 \mathrm{k}$-point mesh and $T=50 \mathrm{~K}$ to determine the leading pairing instability. Working at $T<50 \mathrm{~K}$ requires a much larger $\mathbf{k}$-point mesh, especially when small momenta are important for the pairing.

Figure 3(a) shows the eigenvalues $\lambda$ of Eq. 12 as a function of $U$ for various pairing symmetries at $T=50 \mathrm{~K}$. 
The dominant pairing channel corresponds to a triplet gap with $p$-wave symmetry. The gap function $g_{x}(\mathbf{k})$ for the $p_{x}$-wave state is shown in Fig. 3(b) this state is degenerate with a $p_{y}$-wave state with gap function $g_{y}\left(k_{x}, k_{y}\right)=g_{x}\left(k_{y}, k_{x}\right)$. In order to maximize the gap at the FS, these two states can be combined to form a number of unitary states: (i) $\mathbf{d}(\mathbf{k})=\Delta\left[g_{x}(\mathbf{k}) \pm i g_{y}(\mathbf{k})\right] \hat{\mathbf{e}}_{z}$, (ii) $\mathbf{d}(\mathbf{k})=\Delta\left[g_{x}(\mathbf{k}) \hat{\mathbf{e}}_{x} \pm g_{y}(\mathbf{k}) \hat{\mathbf{e}}_{y}\right]$, and (iii) $\mathbf{d}(\mathbf{k})=$ $\Delta\left[g_{x}(\mathbf{k}) \hat{\mathbf{e}}_{y} \pm g_{y}(\mathbf{k}) \hat{\mathbf{e}}_{x}\right]$. All of these states have the same gap magnitude, shown in Figure 3(c) and are hence degenerate in the present model. The inclusion of additional interactions, e.g. spin-orbit coupling, may lift this degeneracy and favor one state, as in $\mathrm{Sr}_{2} \mathrm{RuO}_{4}{ }^{13}$ The absence of the Knight shift for fields parallel to the $a b$ plane, and the strong out-of-plane anisotropy, suggests that the opposite-spin-pairing $\mathbf{d}(\mathbf{k})=\Delta\left[g_{x}(\mathbf{k}) \pm\right.$ $\left.i g_{y}(\mathbf{k})\right] \hat{\mathbf{e}}_{z}$ state is most likely in LiFeAs 12

The sub-dominant pairing states are a triplet $f$-wave state and a singlet $l=6$ state. The latter can become rather strong close to the IC-AF phase, where the small-q IC fluctuations favor a singlet gap that changes sign over the same small momentum difference. It is interesting to compare our results with the related three-orbital model proposed in Ref. 25, where scattering across the inner hole pocket also produces a similar ring-shaped feature in $\chi^{z z}(\mathbf{q})$. The radius of this hole pocket is much larger than in our model for LiFeAs, however, and there is hence not enough weight at $\mathbf{q}=(0,0)$ to support triplet pairing $[17$ Instead, the larger radius of the ring feature favors slowlyvarying $s$-wave or $B_{1 g}$ singlet gaps. Since triplet $\mathrm{SC}$ is dominant as long as the weight at $\mathbf{q}=(0,0)$ remains high enough down to the critical temperature, we conclude that it will be favored by a small inner hole pocket. Other features of the bands play only a secondary role, e.g. by slightly varying the hopping integrals we can find a much smaller gap on the inner hole pocket, or an almost constant gap on the electron pockets apart from very narrow nodal regions.

\section{CONCLUSIONS}

In this paper we have presented a coherent picture for the properties of LiFeAs driven by magnetic fluctuations. Due to the poor electron-hole nesting and the shallow hole pockets, we find that scattering across the small inner hole pocket dominates the physics, leading to a ring-shaped feature around $(0,0)$ in the momentumdependent magnetic susceptibility. Due to these almost FM processes, triplet $p$-wave superconductivity is favored over singlet pairing.

\section{Acknowledgments}

We would like to thank G. Martins, A. F. Kemper, S. Graser, I. Mazin, S.-H. Baek, H.-J. Grafe, F. Hammerath and B. Büchner for valuable discussions. This research was supported by the DFG under the priority program 1458 (PMRB, CT, and JvdB) and the Emmy-Noether program (MD).
* Electronic address: brydon@theory.phy.tu-dresden.de

$\dagger$ Electronic address: m.daghofer@ifw-dresden.de

1 J. Paglione and R. L. Greene, Nat. Phys. 6, 645 (2010).

2 D. C. Johnston, Adv. Phys. 59, 803 (2010).

3 I. I. Mazin, D. J. Singh, M. D. Johannes, and M. H. Du, Phys. Rev. Lett. 101, 057003 (2008).

4 A. V. Chubukov, D. V. Efremov, and I. Eremin, Phys. Rev. B 78, 134512 (2008).

${ }^{5}$ N. D. Mathur, F. M. Grosche, S. R. Julian, I. R. Walker, D. M. Freye, R. K. W. Haselwimmer, and G. G. Lonzarich, Nature 394, 39 (1998).

6 M. Jourdan, M. Huth, and H. Adrian, Nature 398, 47 (1999).

7 J. H. Tapp, Z. Tang, B. Lv, K. Sasmal, B. Lorenz, P. C. W. Chu, and A. M. Guloy, Phys. Rev. B 78, 060505 (2008).

${ }^{8}$ C. Chu, F. Chen, M. Gooch, A. Guloy, B. Lorenz, B. Lv, K. Sasmal, Z. Tang, J. Tapp, and Y. Xue, Physica C 469, 326 (2009).

9 S. Borisenko, V. Zabolotnyy, D. Evtushinsky, T. Kim, I. Morozov, A. Yaresko, A. Kordyuk, G. Behr, A. Vasiliev, R. Follath, and B. Büchner, Phys. Rev. Lett. 105, 067002 (2010).

10 H. Eschrig and K. Koepernik, Phys. Rev. B 80, 104503 (2009).

11 A. Lankau, K. Koepernik, S. Borisenko, V. Zabolotnyy, B. Büchner, J. van den Brink, and H. Eschrig, Phys. Rev. B
82, 184518 (2010).

12 S.-H. Baek, H.-J. Grafe, F. Hammerath, M. Fuchs, L. Harnagea, S. Wurmehl, J. van den Brink, and B. Büchner, preprint, unpublished.

13 A. P. Mackenzie and Y. Maeno, Rev. Mod. Phys. 75, 657 (2003).

14 S. Graser, T. A. Maier, P. J. Hirschfeld, and D. J. Scalapino, New J. Phys. 11, 025016 (2009).

15 A. Kemper, T. Maier, S. Graser, H.-P. Cheng, P. Hirschfeld, and D. Scalapino, New J. Phys. 12, 073030 (2010).

16 S. Graser, A. F. Kemper, T. A. Maier, H.-P. Cheng, P. J. Hirschfeld, and D. J. Scalapino, Phys. Rev. B 81, 214503 (2010).

17 Q. Luo, G. Martins, D.-X. Yao, M. Daghofer, R. Yu, A. Moreo, and E. Dagotto, Phys. Rev. B 82, 104508 (2010). We verified that the leading triplet channels indeed lie well below the singlet states.

18 Y. Zhou, W.-Q. Chen, and F.-C. Zhang, Phys. Rev. B 78, 064514 (2008).

19 Y. Wan and Q.-H. Wang, EPL 85, 57007 (2009).

20 Z.-H. Wang, H. Tang, Z. Fang, and X. Dai, arXiv:0805.0736 (unpublished).

21 X. Dai, Z. Fang, Y. Zhou, and F.-C. Zhang, Phys. Rev. Lett. 101, 057008 (2008).

22 M. Daghofer, A. Moreo, J. A. Riera, E. Arrigoni, D. J. 
Scalapino, and E. R. Dagotto, Phys. Rev. Lett. 101, 237004 (2008).

${ }^{23}$ G. Xu, W. Ming, Y. Yao, X. Dai, S. Zhang, and Z. Fang, EPL 82, 67002 (2008).

24 P. A. Lee and X.-G. Wen, Phys. Rev. B 78, 144517 (2008).

25 M. Daghofer, A. Nicholson, A. Moreo, and E. Dagotto, Phys. Rev. B 81, 014511 (2010).

26 M. Daghofer, Q.-L. Luo, R. Yu, D. X. Yao, A. Moreo, and E. R. Dagotto, Phys. Rev. B 81, 180514(R) (2010).

27 P. M. R. Brydon, M. Daghofer, and C. Timm, arXiv:1007.1949 (unpublished).

28 Note that unlike Ref. ${ }^{[2]}$ we neglect renormalization of the orbital energies by the Hartree terms.

29 The procedure is similar for the FM phase.

30 Spin fluctuations involving the other pockets are much weaker: a very faint ring corresponding to scattering across the outer hole pocket is only visible at low temperatures, while there is no clear contribution from the electron pockets.

31 D. J. Scalapino, E. Loh, and J. E. Hirsch, Phys. Rev. B 34, 8190 (1986).

32 M. Sigrist and K. Ueda, Rev. Mod. Phys. 63, 239 (1991). 